\title{
Electrospinning of gelatin with tunable fiber morphology from round to flat/ribbon
}

\author{
Fuat Topuz $^{\mathrm{a}, *}$, Tamer Uyar $^{\mathrm{a}, \mathrm{b}, * *}$ \\ a UNAM-National Nanotechnology Research Center, Bilkent University, 06800 Ankara, Turkey \\ b Institute of Materials Science E' Nanotechnology, Bilkent University, 06800 Ankara, Turkey
}

\section{A R T I C L E I N F O}

\section{Article history:}

Received 29 December 2016

Received in revised form 5 May 2017

Accepted 6 June 2017

Available online 7 June 2017

\section{Keywords:}

Gelatin

Nanofibers

Electrospinning

Flat/ribbon fibers

Cross-linking

\begin{abstract}
A B S T R A C T
The electrospinning of gelatin with tunable fiber morphology from round to flat/ribbon was shown, and the detailed studies were conducted to correlate the fiber morphology with electrospinning process parameters and gelatin concentration in electrospinning solution. Particularly, variations in the applied voltage and the concentration of gelatin led to the transition of fiber shape from round to flat/ribbon. The formation of flat-shaped fibers was attributed to rapid evaporation of the solvent (formic acid) from the fiber matrix with increasing the applied voltage and gelatin concentration. On the other hand, round fibers were due to the steady evaporation of formic acid throughout the cross-section of fibers. WAXS analysis revealed that the loss of triple-helical crystalline structure in gelatin after the electrospinning process. The gelatin fibers were cross-linked through treatment with toluene 2,4-diisocyanate (TDI) in a mixed solution of acetone and pyridine, and XPS confirmed the cross-linking of the fibers over an increased carbon content on the elemental composition of the fiber surface due to the incorporated TDI moieties. Overall, this study focuses on morphological tuning of gelatin electrospun fibers towards a flat/ribbon-like structure by variation of electrospinning parameters and polymer concentration, and thus, the proposed concept can be adapted towards flattened/ribbon-like fibers of other protein-based systems by electrospinning.
\end{abstract}

(C) 2017 Elsevier B.V. All rights reserved.

\section{Introduction}

Gelatin is a polyampholyte that is derived from the hydrolysis of native collagen fibers [1,2]. It thus carries many intrinsic characteristics of collagen, including biocompatibility, biodegradability and mechanical strength, through its amino acid composition and peptide mimicry [3]. These distinct advantages of gelatin make it an ideal component to develop proteinaceous constructs of various forms with mechanical strength and durability that are comparable to those of extracellular matrix (ECM) [4-6]. In this context, electrospinning has been validated to be an efficient route to produce nano-/microfibrous materials from numerous synthetic and natural polymers [7] and non-polymeric supramolecular system [8]. In literature, electrospinning of gelatin was also performed to produce fibers from its solutions by using different solvent systems [9-12]. The cross-linking of gelatin fibers could be achieved using various chemicals, including glutaraldehyde [13], genipin [14], D, L-glyceraldehyde [15], or with an exposure to reactive oxygen

\footnotetext{
* Corresponding author.

** Correspondence to: T. Uyar, Institute of Materials Science \& Nanotechnology, Bilkent University, 06800 Ankara, Turkey.

E-mail addresses: fuat.topuz@rwth-aachen.de (F. Topuz), uyar@unam.bilkent.edu.tr (T. Uyar).
}

species, which were generated using a plasma cleaner [15]. Although gelatin preserves many intrinsic characteristics of collagen, it suffers from poor solubility in cold or lukewarm water due to the presence of strong intra- and intermolecular interactions between the polypeptide chains. However, gelatin can be employed after hydration with hot water using particularly designed electrospinning setups, capable of hot-water circulation during the solution feeding [16]. Beyond such particular setups, gelatin was also electrospun using their solutions in acids or binary solvent systems; e.g., Choktaweesap et al. (2007) reported gelatin electrospun fibers using either acetic acid (A.A.) or mixed solvent systems (i.e., A.A./2,2,2-trifluoroethanol (TFE), A.A./dimethyl sulfoxide (DMSO), A.A./ethylene glycol (EG), and A.A./formamide (FA)) [17]. The authors used a fixed voltage at $7.5 \mathrm{kV}$ and varied the concentration of gelatin. They observed the formation of fibers in the concentration range of 21-29\% (w/v). Huang et al. (2004) and Ki et al. (2005) were respectively used TFE and formic acid to generate gelatin fibers with circular cross-sections [11,18].

Electrospinning technique can produce fibers in various shapes and textures, such as uniform, beaded, branched, porous, core-shell, Janus, hollow and flat/ribbon, with dimensions down to nanoscale $[11,19$ 21]. Particularly, the fabrication of flat/ribbon shaped electrospun fibers is a rather challenging task when compared to round fibers. Various parameters like polymer concentration, conductivity of the solutions, and 
the solvent evaporation are involved in the formation of such fiber structures [22,23]. Flat/ribbon-like fibers were previously reported for various polymers, including $\alpha$-elastin polypeptides in water [24], polystyrene in DMF [23], and poly(ether imide) in hexafluoro-2-propanol [23]. Further, gelatin flat-shaped fibers were reported by the electrospinning of gelatin in 2,2,2-trifluoroethanol (TFE) by Rajzer et al. (2014) [25]. However, the authors did not perform any further research to understand the formation of such morphology. In this study, we studied morphological tuning of gelatin fibers towards flat/ribbonlike with variations in the electrospinning process parameters (i.e., applied voltage and flow rate) and gelatin concentration (Fig. 1). Since the gelatin fibers are hydrophilic, and can disintegrate rapidly on contact with water [26], they were also cross-linked through toluene 2,4diisocyanate (TDI) in a mixed solution of acetone and pyridine.

\section{Experimental section}

\subsection{Materials}

Gelatin (from porcine skin, type A), formic acid (>99\%), toluene 2,4diisocyanate (TDI, $95 \%$ ) acetone $(\geq 99.5 \%)$ and pyridine $(\geq 99 \%)$ were purchased from Sigma-Aldrich (Germany). All chemicals were used as received.

\subsection{Electrospinning of gelatin fibers}

Gelatin powder was dissolved in concentrated formic acid (>99\%) under continuous stirring for at least one day. The solutions were loaded into $1 \mathrm{~mL}$ syringes fitted with blunt metallic needles $\left(18 \mathrm{G} \times 1^{1 / 2^{\prime \prime}}\right.$, TERUMO Europe NV). The syringes were placed horizontally on a syringe pump (KDS 101, KD Scientific). The feed rate was varied in the range of $0.83-30 \mu \mathrm{L} / \mathrm{min}$. A high voltage power supply (Matsusada, AU series) was used to apply voltages of several amplitudes (10-22 kV). Randomly oriented fibers were deposited on a grounded stationary rectangular metal collector at $15 \mathrm{~cm}$ distance covered by a piece of aluminum foil. The electrospinning was performed at ca. $25^{\circ} \mathrm{C}( \pm 2)$ in an enclosed Plexiglas chamber. The concentration of gelatin was expressed as $\%(w / v)$.

Gelatin fibers were cross-linked with TDI (57 mM) in a mixed solution of acetone $(10 \mathrm{~mL})$ and pyridine $(1-5 \%(v / v))$ for $2 \mathrm{~h}$. Thereafter, the fibers were rinsed with acetone for several times to remove unbound chemicals from the fiber surface.

\subsection{Rheological analysis}

The viscosity experiments were conducted between the parallel plates of rheometer (Physica MCR 301, Anton Paar) equipped with a Peltier device for temperature control. The upper plate (parallel plate, diameter $25 \mathrm{~mm}$ ) was set at $500 \mu \mathrm{m}$ prior to measurements. The viscosity of gelatin solutions was recorded as a function of shear rate in the range of $0.01-100 \mathrm{~s}^{-1}$. During rheological measurements, a solvent trap was used to prevent the evaporation of formic acid.

\subsection{Dynamic light scattering (DLS)}

Gelatin was treated with concentrated formic acid over time. Within certain time intervals, few microliters were taken from this solution, and diluted with water at a final concentration of $0.1 \%(w / v)$. The sizes of gelatin polypeptides were measured by a photon correlation spectroscopy using a Malvern Nano ZS ZEN3600 (Malvern Instruments Inc., US) at the fixed scattering angle of $173^{\circ}$. Glass cuvettes were used for the measurements in water. The data were analyzed by Zetasizer software (Malvern). The presented data are average values of three measurements. The dynamic light scattering (DLS) measurements give a z-average (or cumulant mean) value, which is an intensity mean and the polydispersity index (PDI). The cumulant analysis has the following form;

$$
\ln \left(g^{(1)}(t)\right)=-\bar{\Gamma} t+\mu_{2} t^{2}+\ldots
$$

where $g^{(1)}$ is the first order correlation function, $\bar{\Gamma}$ is the average decay rate and first cumulant, and $\mu_{2}$ is the second cumulant. The value $\mu_{2} / \bar{\Gamma}^{2}$ is known as PDI.

\subsection{Scanning electron microscopy (SEM)}

The morphology of electrospun gelatin fibers before and after crosslinking was explored with SEM (Quanta 200 FEG, FEI). The mean fiber size $(<D>)$ and their size distributions were calculated by analyzing ca. 100 fibers from SEM images by ImageJ software (NIH, Bethesda, MD, US).

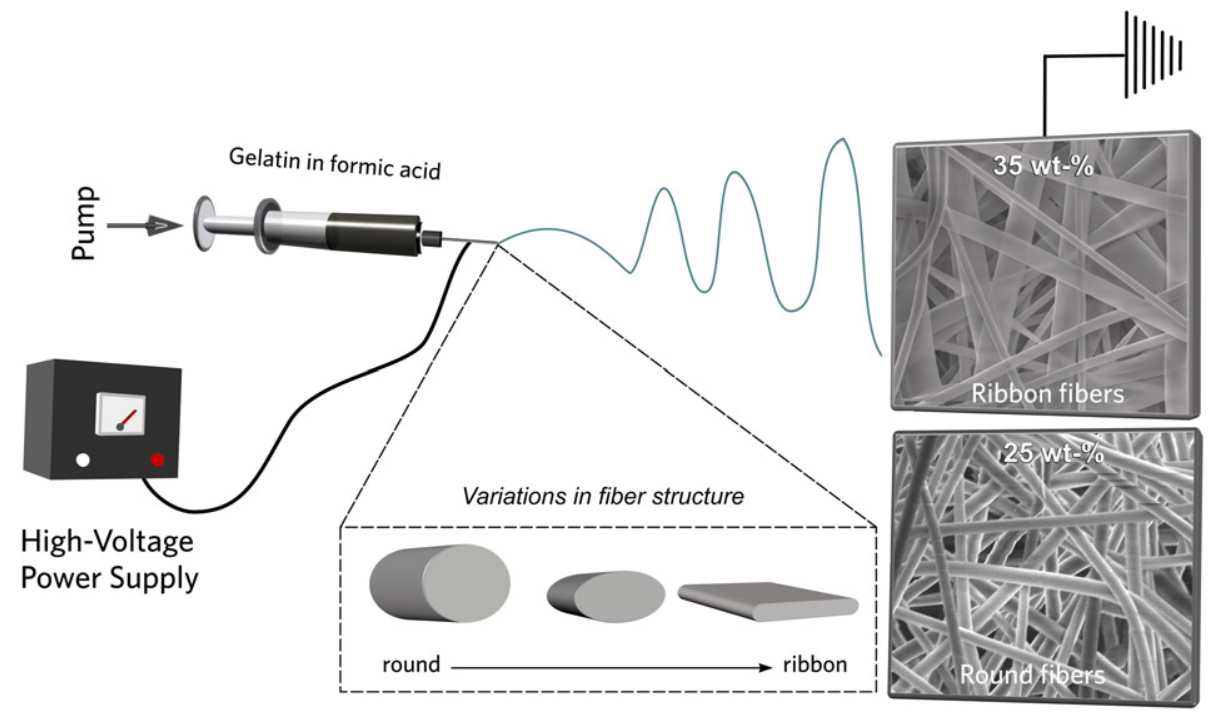

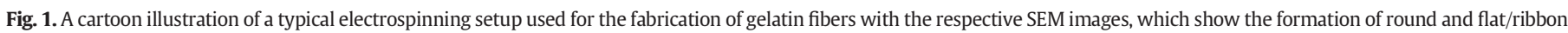
fibers depending on the gelatin concentration used for the electrospinning (25 and 35\% ( $w / v)$, respectively). 


\subsection{Wide-angle X-ray scattering (WAXS)}

WAXS experiments were performed using a PANalytical X'Pert Pro MPD, which was powered by a Philips PW3040/60 X-ray generator and fitted with an X'Celerator detector. X-rays were generated from a $\mathrm{Cu}$ anode supplied with $40 \mathrm{kV}$ and a current of $40 \mathrm{~mA}$. The data were collected over the range of $5-80^{\circ}$ of $2 \theta$ using a scanning X'Celerator detector. All scans were carried out in 'continuous' mode, and the data were later analyzed by PANalytical High Score Plus software (version 2.0).

\subsection{X-ray photoelectron spectroscopy (XPS)}

XPS spectra were recorded by using an X-ray photoelectron spectrometer (Thermo Fisher Scientific, U.K.). As an X-ray source, Al K-alfa X-ray monochromator ( $0.1 \mathrm{eV}$ step size, $12 \mathrm{kV}, 2.5 \mathrm{~mA}$, spot size 400 $\mu \mathrm{m}$ ) was used at an electron take-off angle of $90^{\circ}$ from the sample surface. For all samples, survey spectra were taken 5 times with $50 \mathrm{~ms}$ dwell time (pass energy $200 \mathrm{eV}$ ). All narrow N1s, 01s and C1s spectra were taken 10 times with $50 \mathrm{~ms}$ dwell time (pass energy $30 \mathrm{eV}$ ). The binding scale was referenced to the aliphatic component of $\mathrm{C} 1 \mathrm{~s}$ spectrum at $284.85 \mathrm{eV}$.

\section{Results and discussion}

The viscosities of gelatin solutions were measured as a function of shear rate $(\gamma)$ in a range of $0.01-100 \mathrm{~s}^{-1}$ (Fig. 2a). Fig. 2b shows zeroshear viscosities $\left(\eta^{\circ}\right)$ of the respective gelatin solutions where the solutions with low gelatin concentrations $(<25 \%(w / v))$ have $\eta^{\circ}$ values lower than $2 \mathrm{~Pa} \cdot \mathrm{s}(\sim 20 \mathrm{P})$. On the other hand, at high gelatin concentrations, a significant rise in $\eta^{\circ}$ was observed, suggesting the presence of intra- and intermolecular associations among gelatin chains. Polypeptides are highly sensitive to acidic environments, particularly against strong acids, which cause rapid hydrolysis over the cleavage of proteins into peptide fragments. Weak acids, for example, formic or acetic acids, can also be employed for the digestion of gelatin polypeptides, and in this regard, previous researches on polypeptides showed that the acid-mediated degradation first takes place over aspartic acid residues [27]. Gelatin powder was dissolved in concentrated formic acid, and kept for 9 days. Over time, a significant decrease in the viscosity of gelatin solution was visually observed. Hydrodynamic diameters of gelatin polypeptides were measured by DLS to monitor the degradation process on the molecular scale. Fig. 2(c, d) shows changes in the hydrodynamic diameter $(D)$ of gelatin over time, where slow hydrolysis of the polypeptides during treatment with formic acid was observed. After oneweek exposure to concentrated formic acid, hydrodynamic diameter (D) decreases from ca. 35 to $9 \mathrm{~nm}$, demonstrating rapid hydrolysis of gelatin under acidic conditions. This also implies the significance of the storage time of polypeptides in formic acid prior to the electrospinning; i.e., the electrospinning of polypeptides in acidic solutions will show variations over time. Thus, throughout this article, gelatin solutions were electrospun after one-day exposure to formic acid.

Electrospinning is a process that involves electrical forces to form fibers from a wide range of molecules, including numerous synthetic and natural polymers $[28,29]$, and as well as small molecules like cyclodextrins [8] and cyclodextrin-inclusion complexes [30]. Thus, it is expected that the strength of electrical field should lead to structural variations in electrospun fibers where higher applied voltage leads to thinner fibers due to rapid electrospinning of polymer solution $[7,19]$. In most cases, the electrospinning produces fibers with circular cross-sections, but in some cases, deviations from circular fibers can be observed. Fig. 3 shows the SEM images of the electrospun gelatin nanofibers $\left(c_{\text {gel }}=\right.$

\section{(a)}

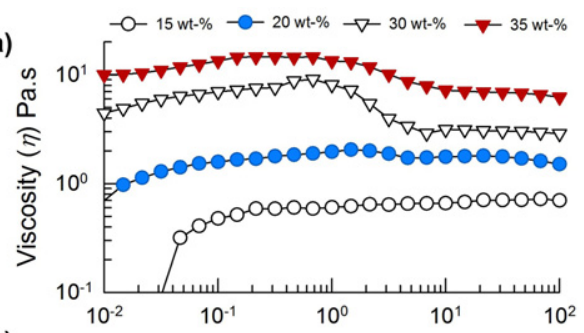

(b)

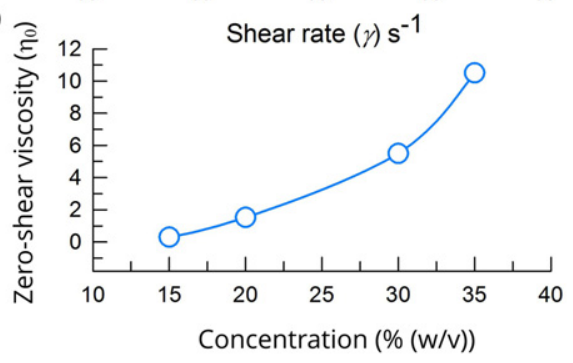

(c)

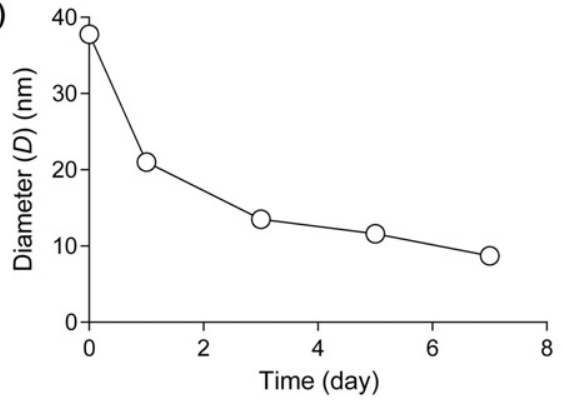

(d)

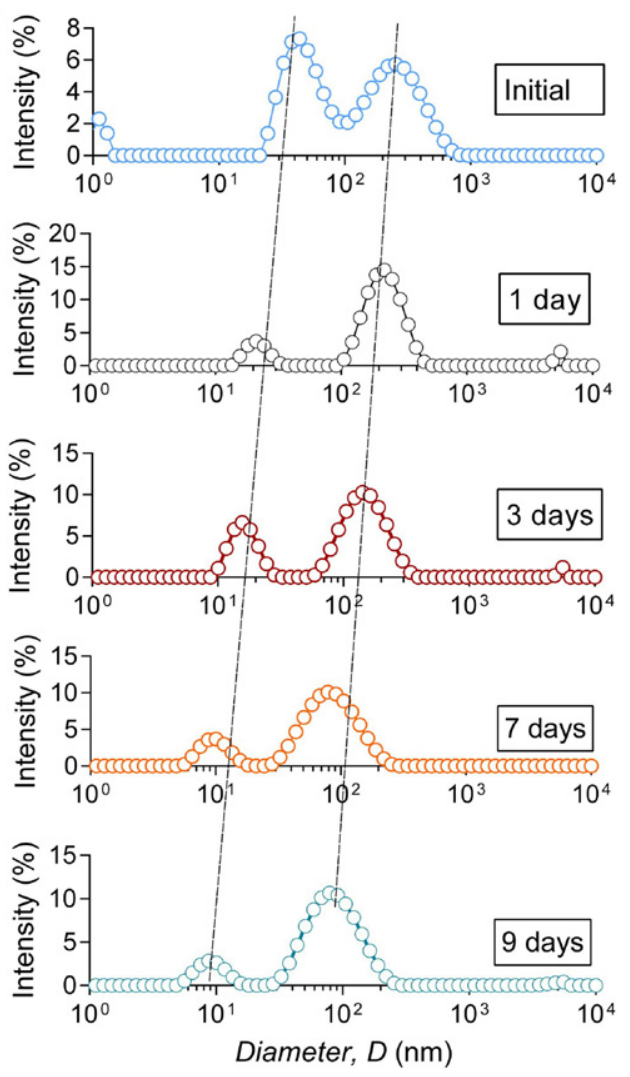

Fig. 2. (a) Viscosity-shear rate profiles and (b) zero-shear viscosities $\left(\eta^{\circ}\right)$ of gelatin solutions in concentrated formic acid. (c) Hydrodynamic diameters $(D)$ of the single-chain polypeptides in formic acid were recorded over 7 days by considering the first peak appeared in the smallest size range. (d) The size-distribution plots of the gelatin polypeptides after exposed to formic acid over time. 


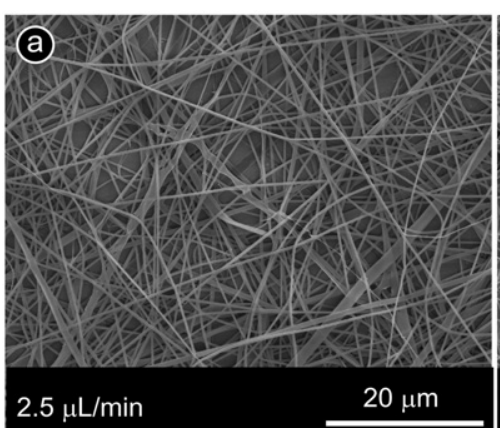

Round fibers

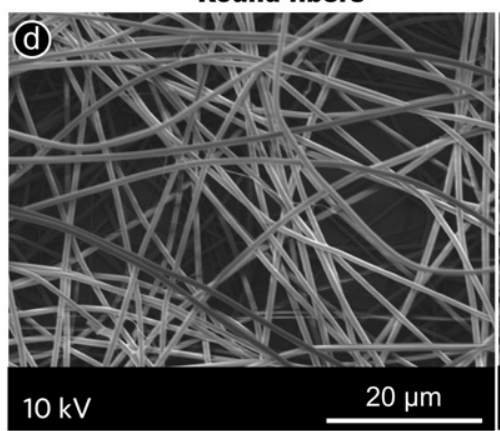

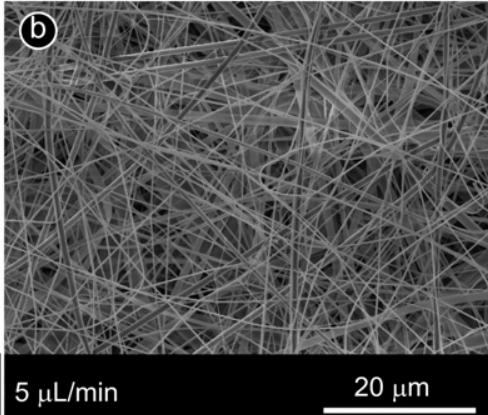

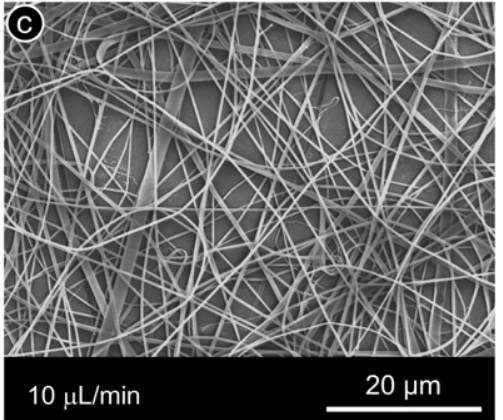

Flat/ribbon fibers
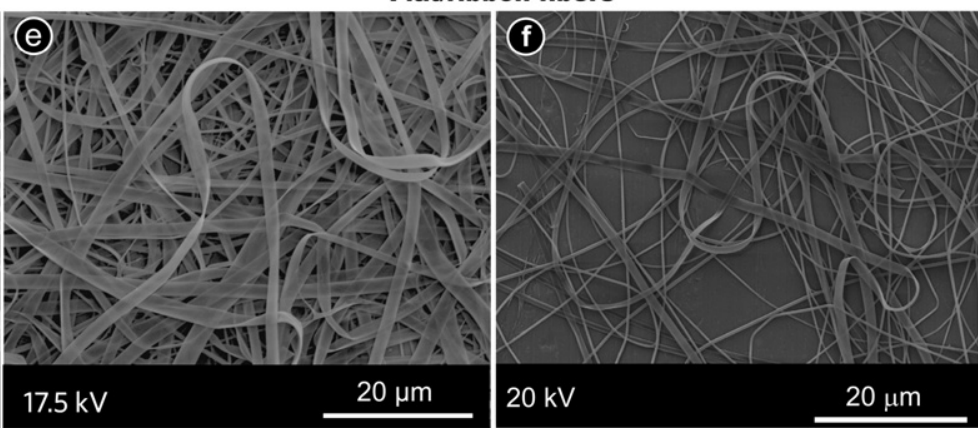

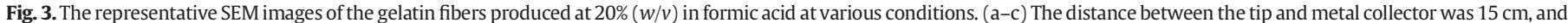

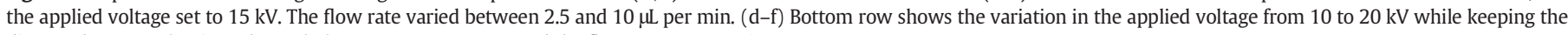
distance between the tip and metal plate constant at $15 \mathrm{~cm}$ and the flow rate at $5 \mu \mathrm{L} / \mathrm{min}$.

$20 \%(w / v))$ at various flow rates $(2.5-10 \mu \mathrm{L} / \mathrm{min})$ and applied voltages $(10-20 \mathrm{kV})$. For all conditions, bead-free nanofibers were produced. However, with increasing the applied voltage from 10 to $20 \mathrm{kV}$, a clear shape transition from round to the flat/ribbon was observed (Fig. 3(df)). On the other hand, variations in the flow rate did not cause such morphological change on the fibers (Fig. 3(a-c)).

During the electrospinning process, the shape and size of a fiber are mainly defined by two major factors; (i) jet ejection from the tip of metal needle once the critical voltage is reached, and (ii) the whipping of jet because of the jet bending instability [31]. In the first stage, the diameter of the jet rapidly decreases, and is followed by the whipping of electrified liquid jet, causing thinner fibers [32,33]. The evaporation of solvent molecules takes place at this stage. Therefore, the amplitude of electrical field affects the formation of gelatin fibers with different shapes, which possibly accelerates the evaporation of solvent molecules and also causes rapid electrospinning of polymer solution [23]. Further, formic acid has rather high conductivity $(5500 \mu \mathrm{S} / \mathrm{cm})$ compared to other weak acids, such as acetic acid, which has conductivity of 318 $\mu \mathrm{S} / \mathrm{cm}$, and as well as much higher than distilled water $(0.04 \mu \mathrm{S} / \mathrm{cm})$ (Table S1, in the Supporting information). The solution conductivity
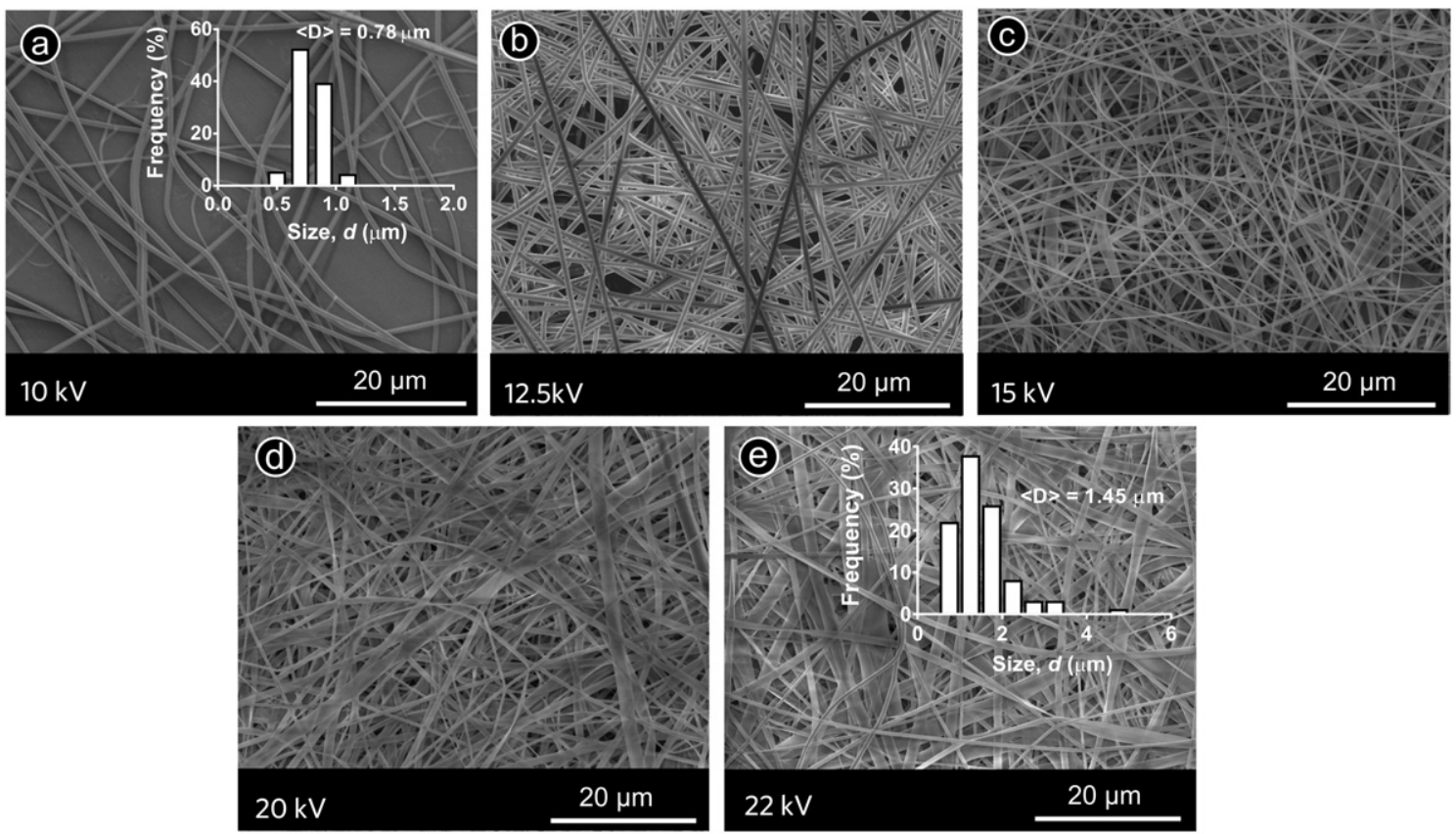

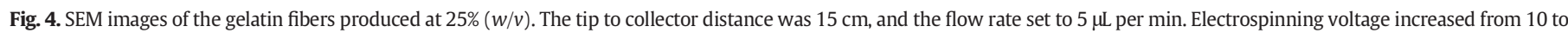
$22 \mathrm{kV}$; (a) $10 \mathrm{kV}$, (b) $12.5 \mathrm{kV}$, (c) $15 \mathrm{kV}$, (d) $20 \mathrm{kV}$ and (e) $22 \mathrm{kV}$. Insets show the size-distribution plots of the respective fibers. 
has a direct effect on the fiber structure where increasing conductivity reduces fiber size [34]. In that sense, high conductivity of formic acid may assist the formation of flat-shaped fibers from gelatin solutions during the electrospinning.

For better understanding the effect of electrical field on fiber morphology, gelatin concentration was increased from 20 to $25 \%(w / v)$ and the applied voltage from 10 to $22 \mathrm{kV}$. Fig. 4 shows SEM images of the fibers with a clear shape transition from round to the flat-like with a voltage rise from 10 to $22 \mathrm{kV}$, where the fiber size increased from $0.78 \mu \mathrm{m}$ to $1.45 \mu \mathrm{m}$ by almost two-time increase in fiber size. Intriguingly, with an increase of the applied voltage, a combination of flat and round-shaped fibers was obtained. This high voltage-driven shape transition is generic for gelatin solutions (in formic acid) as observed for $20 \%$ $(w / v)$ gelatin (Fig. 3(d-f)). The influence of electric field on the flat/ribbon-shaped fiber formation was obvious and could be attributed to rapid electrospinning of gelatin solution [23]. Likewise, flat-shaped Nylon-11 fibers were previously reported at the applied voltage of $20 \mathrm{kV}$ in formic acid [35].

Unlike the applied voltage, the variations in the flow rate did not cause any notable change on the fiber structure. For instance, increasing the flow rate from 2.5 to $30 \mu \mathrm{L}$ per min did not lead to any visible change on the morphology of the fibers produced at 25\% ( $w / v)$ (Fig. 5). This shows that the flow rate does not have any role in the formation of thin/flat-type structure, particularly at low concentrations of gelatin.

The effect of the flow rate at the constant voltage of $20 \mathrm{kV}$ and gelatin concentration at 35\% ( $w / v)$ was investigated through SEM analysis of the electrospun fibers. Fig. 6 shows the SEM images of the gelatin fibers produced at various flow rates $(0.83-5 \mu \mathrm{L} / \mathrm{min})$ where the formation of fibers with flat and round shaped morphology was observed at low flow rates. Whereas, above $5 \mu \mathrm{L} / \mathrm{min}$, flat/ribbon like fibers were dominant.

Gelatin fibers were produced at two different gelatin concentrations (25 and 35\% $(w / v)$ ), where SEM analysis revealed only flat/ribbon-like fibers at high gelatin concentration (Fig. 7). On the other hand, at low gelatin concentration, round-shaped fibers were obtained. Although there is no clear change in the fiber shape by increasing gelatin concentration from 20 to $25 \%(w / v)$, a further concentration increase to $35 \%$ led to the formation of flat-shaped fibers (Fig. 7 (b)). This might be attributed to a substantial increase in viscosity; the zero-shear viscosity $\left(\eta^{\circ}\right)$ of $20 \%(w / v)$ gelatin solution is $2 \mathrm{~Pa} \cdot \mathrm{s}$ while it increases to $11 \mathrm{~Pa} \cdot \mathrm{s}$ for $35 \%$ $(w / v)$ gelatin solution.

The mean size of flat fibers produced at 35\% $(w / v)$ gelatin was measured as $2.2 \mu \mathrm{m}$, and the ratio of axial diameters (long/short) was calculated as high as 7.4 (Fig. 7b, inset). This is much higher than the fibers with circular cross-sections, which should have the corresponding ratio as ca. 1. This ratio of axial diameters (long/short) suggests that the fiber surface is significantly enhanced with a transition from round to flat/ribbon-like. Even though the electrospinning of gelatin in formic acid was previously reported, only fibers with circular cross-sections were observed at low concentration of gelatin (i.e., 7\% $(w / v))$ [18]. In this article, we used very high gelatin concentrations varying between 20 and 35\% $(w / v)$. The most probable pathway for the formation of such fiber structure could be ascribed to rapid evaporation of formic acid during the electrospinning of gelatin solution with increasing the applied voltage and gelatin concentration [23,36]. Rapid jetting of gelatin solution may also assist to the formation of such morphology. The mixed fibers of ribbon and circular were previously reported for aqueous solutions of elastin-like polypeptides, and the authors observed that higher electrospinning voltage led to smaller fibers [37]. Flat-like fiber structure was also reported for Nylon-11 fibers, which were electrospun in formic acid [35]. They observed circular fibers at 10\% $(w / v)$ of the polymer, while the formation of flat-fibers was observed once the concentration was boosted to $20 \%(w / v)$. Koski et al. (2004) has shown a significant effect of the molecular weight on the formation of flat-shaped fibers for poly(vinyl alcohol) (PVA) [38]. Once the molecular weight range for PVA molecules increased from a molecular weight range of $13-23,000$ to $31-50,000 \mathrm{~g} / \mathrm{mol}$, an obvious shift from circular to flat fibers was observed. Similarly, flat-like fibers were also obtained with a concentration increase of PVA. Ghorani and co-workers reported
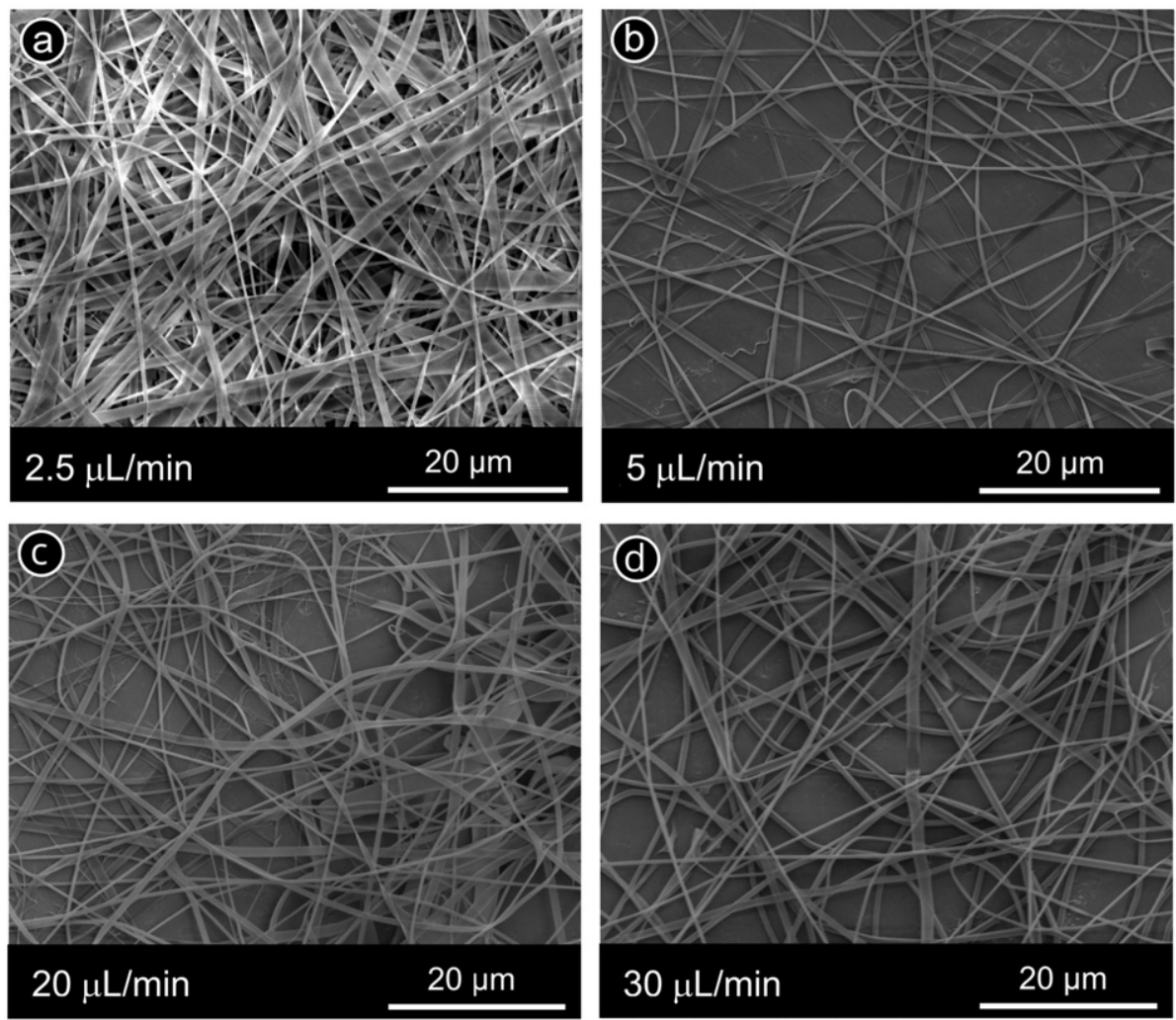

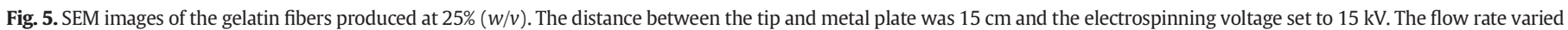
between 2.5 and $30 \mu \mathrm{L} / \mathrm{min}$; (a) $2.5 \mu \mathrm{L} / \mathrm{min}$, (b) $5 \mu \mathrm{L} / \mathrm{min}$, (c) $20 \mu \mathrm{L} / \mathrm{min}$ and (d) $30 \mu \mathrm{L} / \mathrm{min}$. 

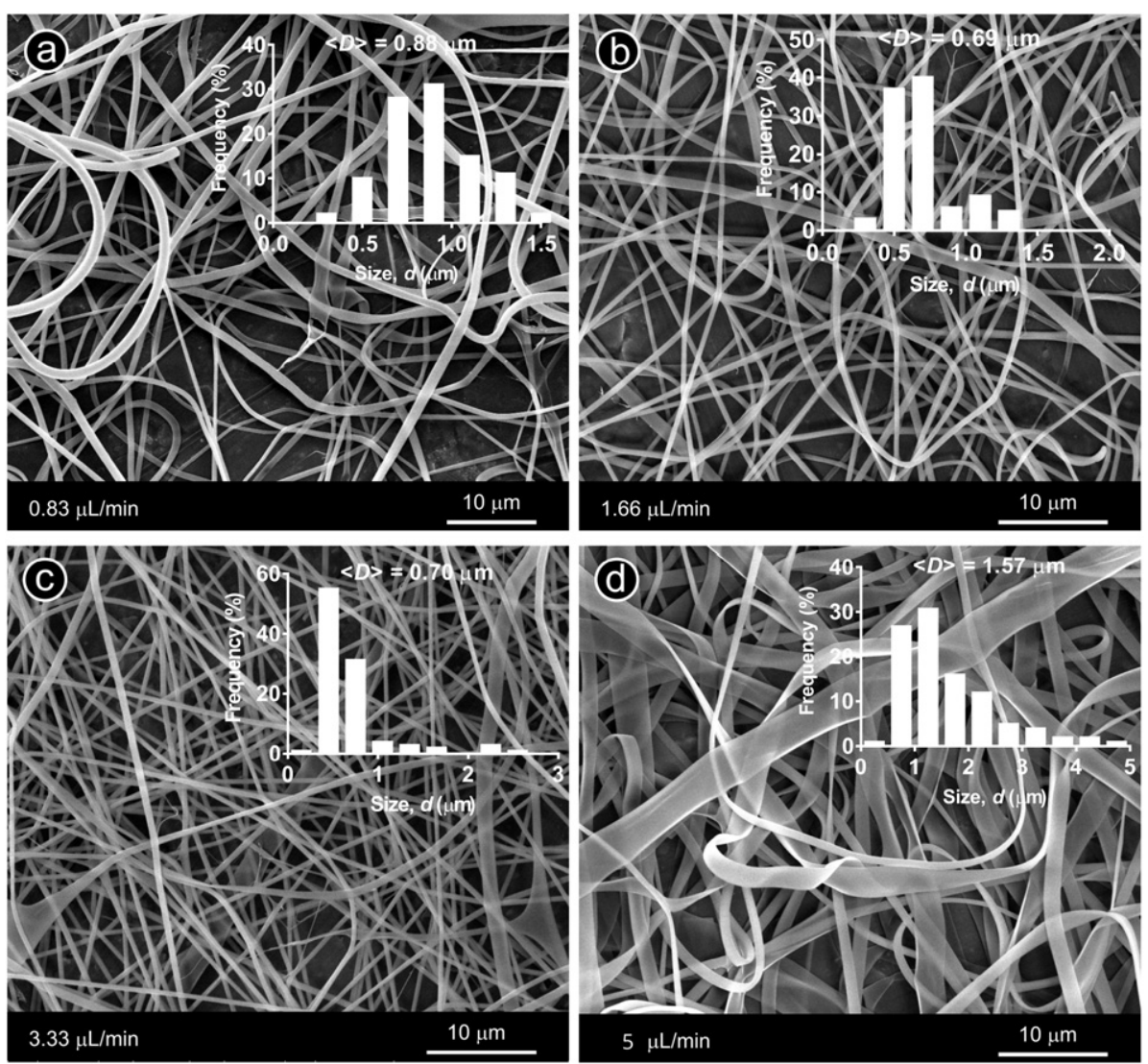

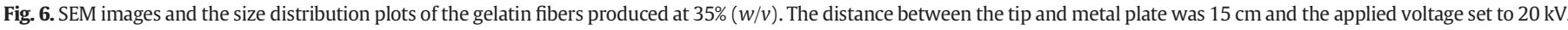
The flow rate varied between 0.83 and $5 \mu \mathrm{L}$ per min.

ribbon-like fiber morphology for cellulose acetate in acetone [39]. The formation of such fiber structure was credited to rapid evaporation of the solvent from the fiber matrix [39]. This in line with the report of Rajzer et al. (2014) on flat type gelatin fibers, in which the formation of such morphology was attributed to rapid evaporation of solvent from the surface of the stream during the electrospinning process [25]. On the other hand, the steady evaporation of formic acid is most likely the cause of the fibers with circular cross-sections (Fig. 7a) [40]. In other words, the homogenous shrinkage of the jet leads to the fibers with circular cross-sections.

The gelatin fibers having flat/ribbon morphology was cross-linked by TDI in acetone solutions having an increasing pyridine concentration.
TDI is a hydrophobic cross-linker with highly reactive free isocyanate groups at both sides, fused with benzene in the middle. Before the addition of TDI linkers, the fibrous webs were first exposed to acetone. SEM images of the gelatin fibers after acetone treatment for one day are shown in Fig. 8a and Fig. S1, where no significant change on the fiber morphology was observed, demonstrating the stability of gelatin fibers in acetone. However, the fibers were partially swollen and turned into round fibers due to the long-term exposure with acetone. Thereafter, TDI linkers at the concentration of $1 \%(w / v)$ and various pyridine concentrations from 1 to $5 \%(v / v)$ was used. Pyridine is generally used to as a catalyst for isocyanate reactions. TDI-based cross-linking in the presence of pyridine occurred very fast, and the fiber structure upon
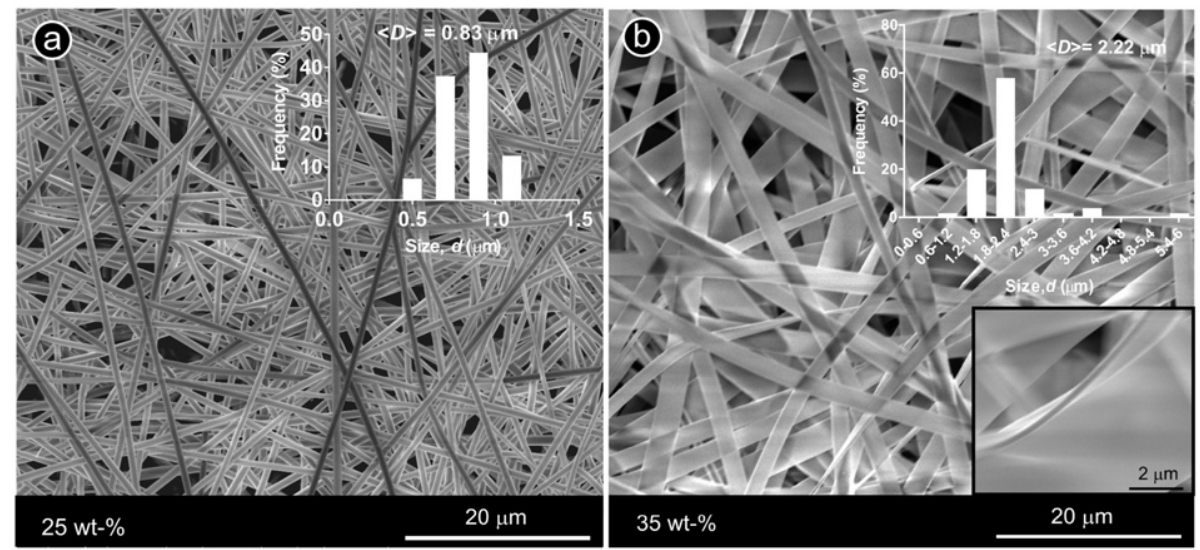

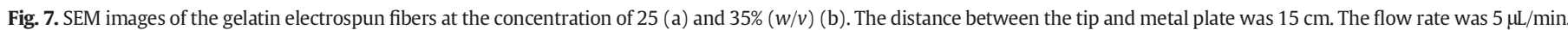
Insets show the size-distribution plots of the fibers, and the SEM image of a flat fiber. 

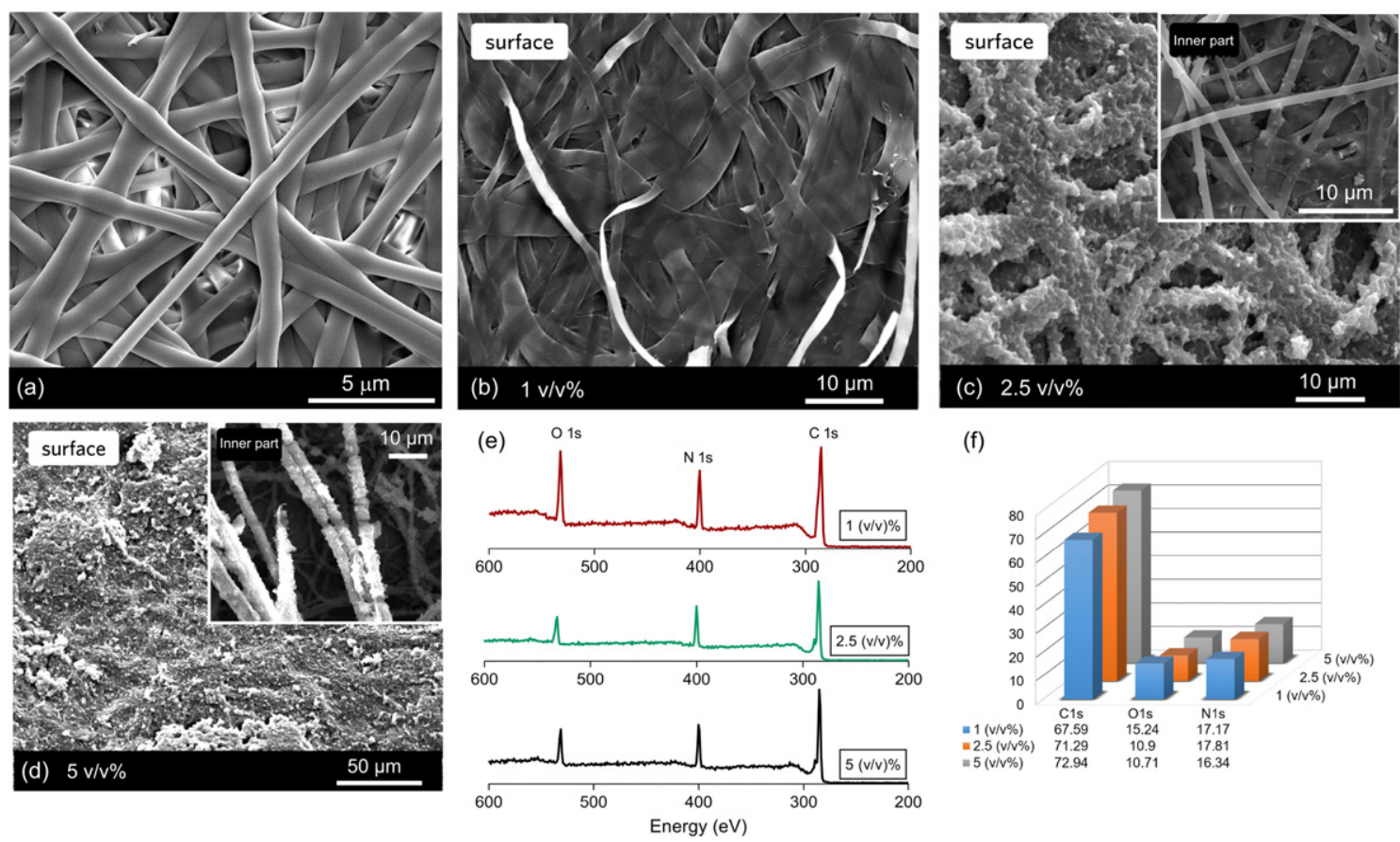

(f)

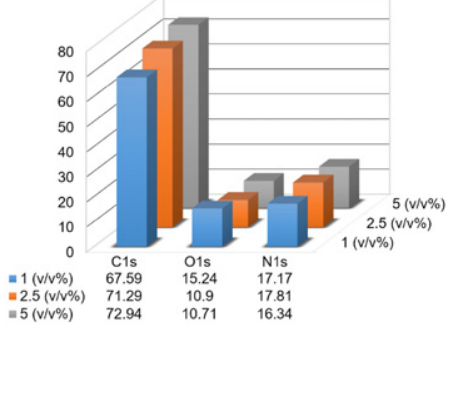

Fig. 8. (a) SEM image of the gelatin fiber one day exposure to acetone. (b-d) SEM images of cross-linked gelatin fibers (i.e., produced at $35 \%(w / v)$, the applied voltage $=20 \mathrm{kV}$ and the flow rate $=5 \mu \mathrm{L} / \mathrm{min})$ at an increasing pyridine content $(\%(v / v))$. Pyridine concentrations; (b) $1 \%(v / v)$, (c) $2.5 \%(v / v)$ and (d) $5 \%(v / v)$. Insets show the SEM images of the inner parts of the fiber mats. (e) XPS survey spectra and (f) the compositional data of the cross-linked fibers.

such cross-linking changed. In Fig. 8(b-d), the SEM images of the fiber after having cross-linked with TDI clearly demonstrated a transition from the fibrous network to the bulk-material form with an increasing pyridine content. Although the inner-parts of the mats displayed a fibrous matrix, the solvent-contact surfaces lost the fiber structure due to excessive cross-linking and instant swelling. Increasing pyridine content led to significant changes on the fiber texture by forming nanoaggregates on the fibers. This was due that the TDI linkers forms aggregates in the solvent mixture, where pyridine catalyzed crosslinking reactions towards nanoaggregates on the fiber surface.

For the determination of cross-linking efficiency, XPS was used to elucidate the chemical compositions of the surfaces of the cross-linked fibers. Fig. 8(e) shows the XPS survey spectra of the cross-linked fibers by TDI in acetone at various pyridine concentrations. It is normally expected that the high degree of the cross-linking should increase carbon content on the chemical composition. With increasing pyridine concentration, $\mathrm{C} 1 \mathrm{~s}$ peak becomes more dominant compared to the 01s peak, suggesting more TDI moieties bound on the fibers. Fig. 8(f) shows the elemental compositions of the fiber surfaces where the $C$ ratio boosted from 67.5 to $73 \%$ with increasing pyridine concentration from 1 to $5 \%$ $(v / v)$.

The structural analysis of gelatin fibers before and after cross-linking was explored through wide-angle X-ray scattering (WAXS), where crystalline zones display sharp diffraction peaks and amorphous regions show broader ones. The gelatin, a hydrolyzed collagen, has some inherited crystalline segments of collagen, particularly at $\sim 20.40^{\circ}$ ( $d$ spacing: $0.43 \mathrm{~nm}$ ) and $8.35^{\circ}$ ( $d$-spacing: $1.57 \mathrm{~nm}$ ) because of the triple-helical crystalline structure of collagen renatured in gelatin (Fig. 9) [41]. During the electrospinning process, intramolecular associations were damaged to some extent, which induce broader and smaller diffraction peaks. The structural arrangements that are driven by intraand intermolecular interactions in gelatin polypeptides decreased during the electrospinning so that broad peaks at $20^{\circ}$ and $8.35^{\circ}$ became nearly invisible in the corresponding ranges, and the material becomes amorphous.

\section{Conclusion}

This study describes the electrospinning of gelatin solutions with tunable fiber morphology from round to flat/ribbon. The formation of flat/ribbon fiber structure was attributed to the applied voltage and gelatin concentration for the electrospinning. For instance, by increasing the applied voltage from 10 to $25 \mathrm{kV}$ in the electrospinning process, the formation of flat/ribbon-type fibers was clearly observed. During the electrospinning process, rapid release of formic acid at high voltages and gelatin concentrations might led to flat/ribbon-like electrospun gelatin fibers. Whereas, the formation of fibers with circular cross-sections can be attributed to the steady evaporation of formic acid (i.e., homogenous shrinkage of the jet) from the fiber matrix. WAXS analysis

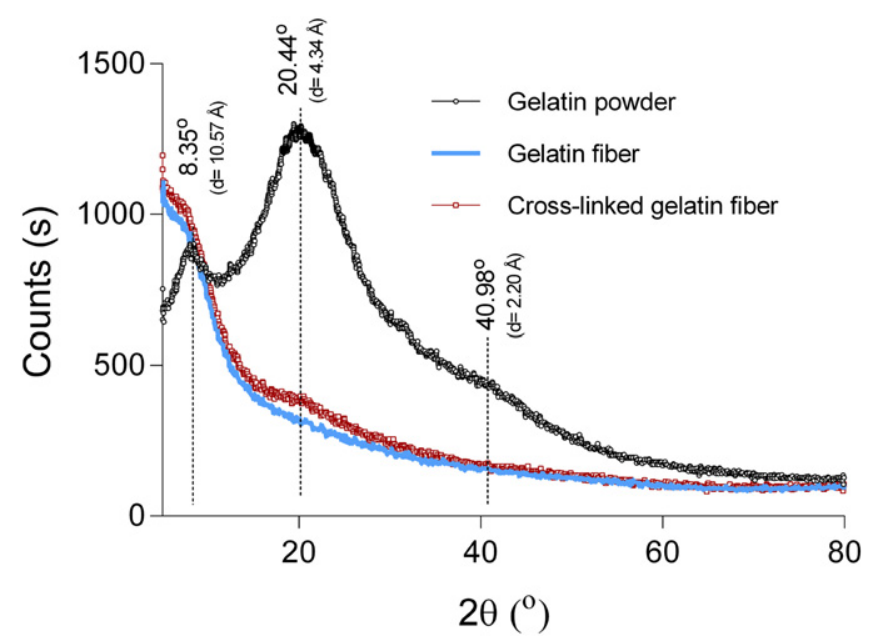

Fig. 9. Wide-angle XRD patterns of the flat-shaped gelatin fibrous mat (i.e., produced at $35 \%(w / v)$, the applied voltage $=20 \mathrm{kV}$ and the flow rate $=5 \mu \mathrm{L} / \mathrm{min})$ and gelatin powder. Significant peaks were shown with corresponding $d$-spacing values. 
revealed that the electrospinning of gelatin led to structural changes in gelatin polypeptides. The fibers were cross-linked by diisocyanate linkers (TDI) in a mixed solution of acetone and pyridine. This crosslinking route was highly efficient and led to highly cross-linked fiber mats. However, the fiber morphology was greatly influenced by the used pyridine content. XPS analysis demonstrated the cross-linking of the fibers with an increased carbon content of the fibers. Overall the paper reports an initiative example of tuning of the fiber structure of a polypeptide, gelatin as an example, and has potential applications for other protein-based systems.

\section{Acknowledgements}

T. U. acknowledges the partial support from Turkish Academy of Sciences - Outstanding Young Scientists Award Program (TUBA-GEBIP). F. T. thanks the TUBITAK Co-Funded Brain Circulation Scheme (project number: 116C031).

\section{Appendix A. Supplementary data}

Supplementary data to this article can be found online at http://dx. doi.org/10.1016/j.msec.2017.06.001.

\section{References}

[1] M.C. Gómez-Guillén, B. Giménez, M.E. López-Caballero, M.P. Montero, Functional and bioactive properties of collagen and gelatin from alternative sources: a review, Food Hydrocoll. 25 (2011) 1813-1827.

[2] K.B. Djagny, Z. Wang, S. Xu, Gelatin: a valuable protein for food and pharmaceutical industries: review, Crit. Rev. Food Sci. Nutr. 41 (2001) 481-492.

[3] A. Veis, The physical chemistry of gelatin, Int. Rev. Connect. Tissue Res. 3 (1965) 113-200.

[4] J.-Y. Lai, Biocompatibility of chemically cross-linked gelatin hydrogels for ophthalmic use, J. Mater. Sci. Mater. Med. 21 (2010) 1899-1911.

[5] S. Nagarajan, L. Soussan, M. Bechelany, C. Teyssier, V. Cavailles, C. Pochat-Bohatier, P. Miele, N. Kalkura, J.-M. Janot, S. Balme, Novel biocompatible electrospun gelatin fiber mats with antibiotic drug delivery properties, J. Mater. Chem. B 4 (2016) 1134-1141.

[6] K.R. Stevens, N.J. Einerson, J.A. Burmania, W.J. Kao, In vivo biocompatibility of gelatin-based hydrogels and interpenetrating networks, J. Biomater. Sci. Polym. Ed. 13 (2002) 1353-1366.

[7] T. Uyar, E. Kny, Electrospun Materials for Tissue Engineering and Biomedical Applications: Research, Design and Commercialization, Elsevier, Woodhead Publishing Series in Biomaterials, June 2017 ISBN: 9780081010228.

[8] A. Celebioglu, T. Uyar, Electrospinning of nanofibers from non-polymeric systems: polymer-free nanofibers from cyclodextrin derivatives, Nanoscale 4 (2012) 621-631.

[9] M. Erencia, F. Cano, J.A. Tornero, M.M. Fernandes, T. Tzanov, J. Macanás, F. Carrillo, Electrospinning of gelatin fibers using solutions with low acetic acid concentration: effect of solvent composition on both diameter of electrospun fibers and cytotoxicity, J. Appl. Polym. Sci. 132 (2015) 42115.

[10] M. Li, Y. Guo, Y. Wei, A.G. MacDiarmid, P.I. Lelkes, Electrospinning polyanilinecontained gelatin nanofibers for tissue engineering applications, Biomaterials 27 (2006) 2705-2715.

[11] Z.-M. Huang, Y.Z. Zhang, S. Ramakrishna, C.T. Lim, Electrospinning and mechanical characterization of gelatin nanofibers, Polymer 45 (2004) 5361-5368.

[12] J.-H. Song, H.-E. Kim, H.-W. Kim, Production of electrospun gelatin nanofiber by water-based co-solvent approach, J. Mater. Sci. Mater. Med. 19 (2008) 95-102.

[13] W. Lu, M. Ma, H. Xu, B. Zhang, X. Cao, Y. Guo, Gelatin nanofibers prepared by spiralelectrospinning and cross-linked by vapor and liquid-phase glutaraldehyde, Mater. Lett. 140 (2015) 1-4.

[14] S. Panzavolta, M. Gioffrè, M.L. Focarete, C. Gualandi, L. Foroni, A. Bigi, Electrospun gelatin nanofibers: optimization of genipin cross-linking to preserve fiber morphology after exposure to water, Acta Biomater. 7 (2011) 1702-1709.
[15] K. Sisson, C. Zhang, M.C. Farach-Carson, D.B. Chase, J.F. Rabolt, Evaluation of crosslinking methods for electrospun gelatin on cell growth and viability, Biomacromolecules 10 (2009) 1675-1680.

[16] S. Ostrovidov, X. Shi, L. Zhang, X. Liang, S.B. Kim, T. Fujie, M. Ramalingam, M. Chen, K. Nakajima, F. Al-Hazmi, H. Bae, A. Memic, A. Khademhosseini, Myotube formation on gelatin nanofibers - multi-walled carbon nanotubes hybrid scaffolds, Biomaterials 35 (2014) 6268-6277.

[17] N. Choktaweesap, K. Arayanarakul, D. Aht-ong, C. Meechaisue, P. Supaphol, Electrospun gelatin fibers: effect of solvent system on morphology and fiber diameters, Polym. J. 39 (2007) 622-631.

[18] C.S. Ki, D.H. Baek, K.D. Gang, K.H. Lee, I.C. Um, Y.H. Park, Characterization of gelatin nanofiber prepared from gelatin-formic acid solution, Polymer 46 (2005) 5094-5102.

[19] J.A. Matthews, G.E. Wnek, D.G. Simpson, G.L. Bowlin, Electrospinning of collagen nanofibers, Biomacromolecules 3 (2002) 232-238.

[20] S. Khorshidi, A. Solouk, H. Mirzadeh, S. Mazinani, J.M. Lagaron, S. Sharifi, S. Ramakrishna, A review of key challenges of electrospun scaffolds for tissue-engineering applications, J. Tissue Eng. Regen. Med. 10 (9) (2016) 715-738.

[21] S. Ramakrishna, K. Fujihara, W.E. Teo, T.C. Lim, Z. Ma, An Introduction to Electrospinning and Nanofibers, 2005.

[22] A. Frenot, I.S. Chronakis, Polymer nanofibers assembled by electrospinning, Curr. Opin. Colloid Interface Sci. 8 (2003) 64-75.

[23] S. Koombhongse, W. Liu, D.H. Reneker, Flat polymer ribbons and other shapes by electrospinning, J. Polym. Sci. B Polym. Phys. 39 (2001) 2598-2606.

[24] J. Araujo, J. Padrão, J.P. Silva, F. Dourado, D.M. Correia, G. Botelho, J.L. Gomez Ribelles, S. Lanceros-Méndez, V. Sencadas, Processing and characterization of $\alpha$-elastin electrospun membranes, Appl. Phys. A 115 (2014) 1291-1298.

[25] I. Rajzer, E. Menaszek, R. Kwiatkowski, J.A. Planell, O. Castano, Electrospun gelatin/ poly(E-caprolactone) fibrous scaffold modified with calcium phosphate for bone tissue engineering, Mater. Sci. Eng. C 44 (2014) 183-190.

[26] Y.Z. Zhang, J. Venugopal, Z.M. Huang, C.T. Lim, S. Ramakrishna, Crosslinking of the electrospun gelatin nanofibers, Polymer 47 (2006) 2911-2917.

[27] R.A. Kenley, N.W. Warne, Acid-catalyzed peptide bond hydrolysis of recombinant human interleukin 11, Pharm. Res. 11 (1994) 72-76.

[28] S. Agarwal, A. Greiner, J.H. Wendorff, Electrospinning of manmade and biopolymer nanofibers-progress in techniques, Materials, and Applications, Advanced Functional Materials 19 (2009) 2863-2879.

[29] S. Agarwal, J.H. Wendorff, A. Greiner, Use of electrospinning technique for biomedical applications, Polymer 49 (2008) 5603-5621.

[30] A. Celebioglu, F. Kayaci-Senirmak, S. Ipek, E. Durgun, T. Uyar, Polymer-free nanofibers from vanillin/cyclodextrin inclusion complexes: high thermal stability, enhanced solubility and antioxidant property, Food Funct. 7 (2016) 3141-3153.

[31] C. Wang, Y.-W. Cheng, C.-H. Hsu, H.-S. Chien, S.-Y. Tsou, How to manipulate the electrospinning jet with controlled properties to obtain uniform fibers with the smallest diameter?-a brief discussion of solution electrospinning process, J. Polym. Res. 18 (2011) 111-123.

[32] Y.M. Shin, M.M. Hohman, M.P. Brenner, G.C. Rutledge, Experimental characterization of electrospinning: the electrically forced jet and instabilities, Polymer 42 (2001) 09955-09967.

[33] D.H. Reneker, A.L. Yarin, H. Fong S. Koombhongse, Bending instability of electrically charged liquid jets of polymer solutions in electrospinning, J. Appl. Phys. 87 (2000) 4531-4547.

[34] T. Uyar, F. Besenbacher, Electrospinning of uniform polystyrene fibers: the effect of solvent conductivity, Polymer 49 (2008) 5336-5343.

[35] M. Dhanalakshmi, J.P. Jog, Preparation and characterization of electrospun fibers of Nylon 11, Express Polym Lett 2 (2008) 540-545.

[36] V. Pillay, C. Dott, Y.E. Choonara, C. Tyagi, L. Tomar, P. Kumar, L.C. du Toit, V.M.K. Ndesendo, A review of the effect of processing variables on the fabrication of electrospun nanofibers for drug delivery applications, J. Nanomater. 2013 (2013) 22.

[37] Y. Ner, J.A. Stuart, G. Whited, G.A. Sotzing, Electrospinning nanoribbons of a bioengineered silk-elastin-like protein (SELP) from water, Polymer 50 (2009) 5828-5836.

[38] A. Koski, K. Yim, S. Shivkumar, Effect of molecular weight on fibrous PVA produced by electrospinning, Mater. Lett. 58 (2004) 493-497.

[39] B. Ghorani, S.J. Russell, P. Goswami, Controlled morphology and mechanical characterisation of electrospun cellulose acetate fibre webs, International Journal of Polymer Science 2013 (2013) 12.

[40] D.H. Reneker, A.L. Yarin, Electrospinning jets and polymer nanofibers, Polymer 49 (2008) 2387-2425.

[41] J.K.D. Naskar, S.C. Kundu, N.R. James, Fabrication of cationized gelatin nanofibers by electrospinning for tissue regeneration, RSC Adv. 5 (2015) 89521-89530. 